\title{
Wireless Network and Communication in Smart Grid Technology
}

\author{
Shardul Pathak ${ }^{1}$, Sagar Majgude ${ }^{1}$, Sagar Maske $^{1}$, Aneesh Sakure $^{1}$, \\ Shakambhari Pathak ${ }^{2}$ \\ ${ }^{I}$ B. Tech Computer Science Engineering, Vishwakarma Institute of Information \\ Technology, Maharashtra, India \\ ${ }^{2}$ Analyst Trainee, Baxter Innovations and Business Solutions Pvt. Ltd.
}

\begin{abstract}
In this century where everyone is talking about making machine smart, intelligent, it is need of technology to go two steps ahead and make smarter systems upon which humans can rely. Smart Grid is nothing but making the systems at home intelligent so as to make life easy without spending lot of money. This paper will help you understand what actually smart grid is, how it can be implemented and mainly focus upon the wireless communication in Smart Grid. The paper will present the architecture of Smart Grid using wireless connectivity and explain the importance of Home Area Networks. Security is the measure concern in case of wireless communication which is explained with some of the protocols to be used and Methods to avoid security threats. Wireless Technologies like Wi-Max, Wi-Fi, Zig-Bee and their use in the Smart Grid architecture is covered. Today, we are searching for a solution with less efforts and maintenance required and for the complicated electrical system, Smart Grid is the best solution to be implemented. Conventional Grid could not solve the problem of peak loads where users/consumers were unable to receive a proper service, it is complicated for the central system to manage large amount of load alone. Smart Grid solves this problem by distributing the management of energy and how it does, we will see in some of the chapters in this report. Smart devices like smart meters, Electric Vehicles can be used to make homes smarter. Smart Grid technology does not solve the problem of electricity only but also for water, gas etc. This technology is most suitable for smart city/village projects helping in making smarter devices and using communication between the devices, making human life more reliable and comfortable.
\end{abstract}

Keywords: Inventory Management System, barcode scanner, update, tracking inventory, time saving. 


\section{Introduction to Smart Grid}

\subsection{What is Smart Grid:}

A grid is a network of generators, distributors, controllers and consumers of electricity. A traditional grid (Conventional Grid) is a system in which electricity is produced at one end and is distributed amongst the consumers. A traditional grid is a one-way transmission system in which electricity is generated and transmitted and consumed by the consumer. Unlike the traditional grid a Smart Grid is a network of generators, distributors, controllers and consumers using two-way communication. In a Smart Grid, the concept of electricity distribution is not restricted till transmission, it is the network which provides us with twoway data and information transfer. Unlike the communication network smart grid does not only route the packets of data and information but it also routes the power flows having many constraints. The power flow in Smart Grid is two way that is the user can also generate electricity at home and put back to network. Example of this can be electricity produced at home using solar panels. The communication in Smart Grid is digital (real-time). It is a grid which is self-monitored, self-healed. The basic concept of Smart Grid is making the traditional grid more reliable and "SMART"

\subsection{NIST Definition and Model of Smart Grid:}

Many definitions of Smart Grid have derived till now. The National Institute for Standards and Technology gives the definition of Smart Grid as:

It is called as "electricity with a brain" or "energy internet" and "the electro net".

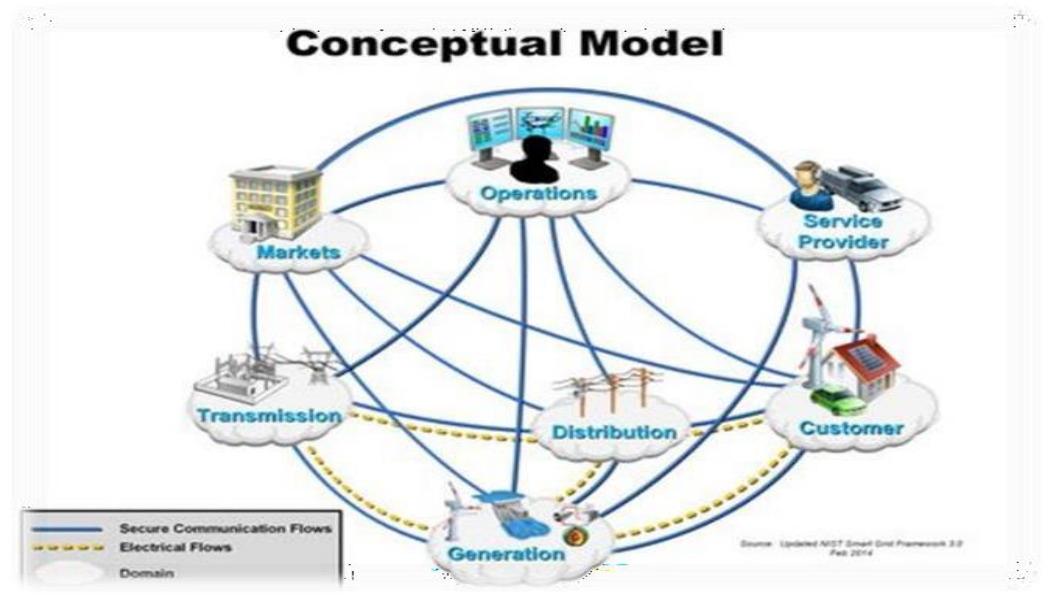

Figure 1.1 Model of Smart Grid

\subsection{Advantages of Smart Grid over Traditional Grid:}

Smart Grid is not just about creating advance intelligent grid. Smart Grid will help the electricity distribution to be intelligent, more secure, reliable. It will also help in saving money by using techniques like advance metering, demand-response use of electricity, managing the peak load, efficient power networks, managing the energy consumptions by detecting the time period when electricity will be consumed at the highest rate to lowest 
rate. These managements cannot be done in the conventional grids. Also, the smart grid ensures that renewable power sources like wind farms, solar plants and hydro stations can be integrated which will help in managing the three fields. Smart grid will help in making intelligent energy consumption. It will also help in using the renewable energy sources effectively. Smart Grid will help in taking IoT to the next level by using smarter energy consumption techniques.

\subsection{Application of Smart Grid Technology:}

Implementation of Digital Technology in Smart Grids enables the reliability, efficiency and availability to the user regarding all the things which makes a step towards the economic stability of the country. Right at the start of transition time it become difficult to execute testing, to improve the technology by upgradation, developing and maintaining standards on a standard level and also application of these intelligent grids solve all above problems. Applications of Smart Grid are: 1.They improve the adeptness of transmission lines. 2. Electric Vehicles. 3.Smart City. 4. Home Energy Management System. 5. Quick recovery after any immediate damage/disturbance in lines and feeders. 6. Reduction of peak demand. 7. They possess the power to be integrated with renewable energy sources which leads to sharing of the load and reduction of the load on large scale.

\section{Wireless Networks and Communication}

\subsection{Types of networks according to smart grid:}

The communication in Smart Grid needs to be effective so creating a multi-layer architecture would be better for effective communication. Different types of networks can be used to create the effective multilayer architecture. Wireless Local Area Networks can be implemented in Smart Grids which are a better option than other wired or wireless networks. Home Area Network. Neighbourhood Area Network. Wide Area Network. Substation Area Network can be used in the multi-layer architecture. A Wide Area Network will be used to connect the electricity generators covering larger areas. WAN is used to connect multiple NANs.

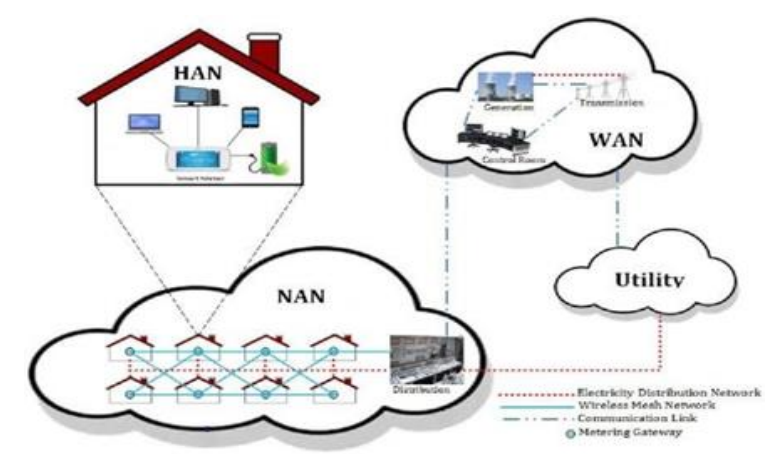

Figure 2.1 Types of Network in Smart Grid Model

\subsection{Wireless Topologies for Smart Grid:}

Wireless Local Area Networks can be implemented using Mesh Topology which is effective in case of Smart Grid networking architecture. Mesh Topology has many advantages like self-healing, easy to incorporate the new device in the network, end to end 
communication making it easy to transfer information in both the directions also scalability is the main feature of Mesh Topology. Mesh Topology is a structure in which every node is connected to multiple or all nodes in the network. Mesh Topology can be of two types: 1)Full Mesh Topology - Here, each node in the network is connected to every other node in the network.

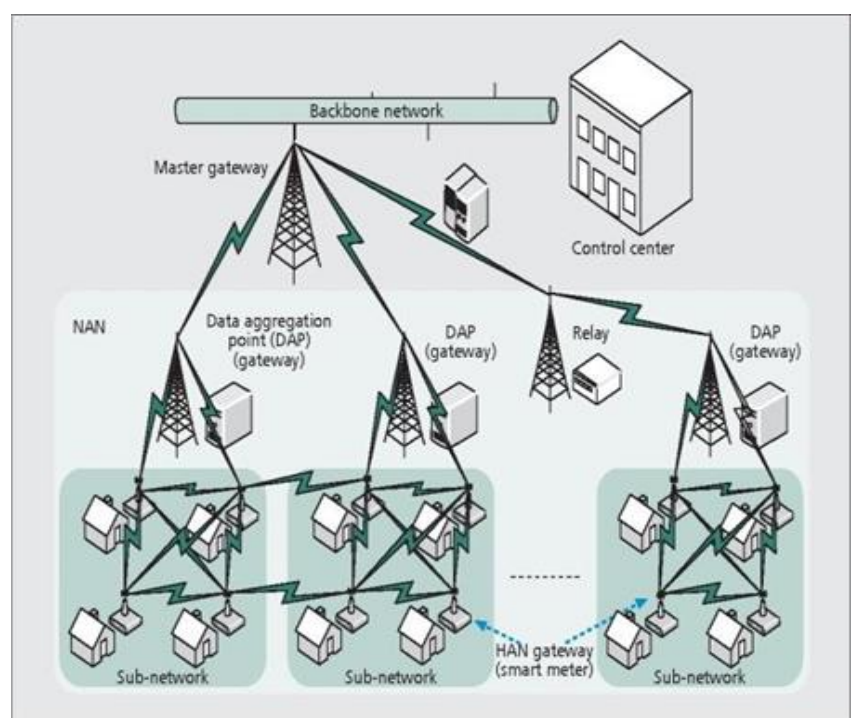

Figure 2.2 Mesh Topology

2)Partial Mesh Topology - In this type, there are at least two nodes in the topology which are connected to multiple nodes in the network Mesh Topology has an advantage that even if one connection fails, other can be used to transfer the data. It is the best suitable topology in data transfer traffic. In Mesh distributed nodes data is transferred by hopping from node to node until the receiver is not reached or the mesh gateway is not reached. Mesh Topology has many disadvantages: 1 .It is expensive than other topologies. 2. The cost of maintenance is very high. 3.Building the topology and managing it is complicated. 4. Mesh topology can have many chances of redundant data connection which reduces the efficiency of the network.

\section{Implementing Smart Grid}

\subsection{Remote Sensing:}

Remote sensing means obtaining information about any object without actually coming in contact with the object. Different sensors are used for this technique. There are two types of sensors: 1)Active Sensors - These sensors use their own light source for detecting the objects. The light emitted by the active sensor is reflected by the object and using that light the sensor detects the object. Laser Altimeter which is a device used to measure the height of a platform (spacecraft or aircraft) above the surface. Radar is active radio detection and ranging sensor that has its own source of electromagnetic energy. Scatterometer, Sounder are some of the other examples of active sensors. 2)Passive Sensors - These sensors use the natural source of light as sunlight to detect the objects. These sensors do not have their own light source for detecting the object. Accelerometer (A device detecting change in velocity), Radiometer (A device able to detect hundreds of very narrow spectral bands), Imaging Radiometer, Spectroradiometer are some of the examples of passive sensors. Sensors are another technique of wireless technology used in Smart Grid which is helpful in Fault Detection and detecting black-outs, Scalability of the network and managing the network 
smartly also sensors are useful in smart communication bridging the gap of communication between devices. It also enables better utilization of the grid by detecting the device's efficiency at a particular time. Remote sensing is one of the important wireless measures of Smart Grid Applications.

\subsection{Automated Metering:}

Automated Metering is the concept which talks about using intelligent meters capable of interacting with the other devices and giving information about the consumption of the electricity, detecting the faults and managing the loads. Smart billing is possible due to automated metering. Wireless techniques as Wi-Fi, ZigBee, Wi-SUN are used for smart meters. Two-way communication channel is necessary to support readings and proactive actions. Automatic Meter Reading increases the Transparency for the user. The wireless devices do the essential job of transmission of data between the meter and the display device. Following diagram shows a basic architecture of the automated metering system in which the wireless module behaves as an intermediate system for communication. The data transferred is in digital form.

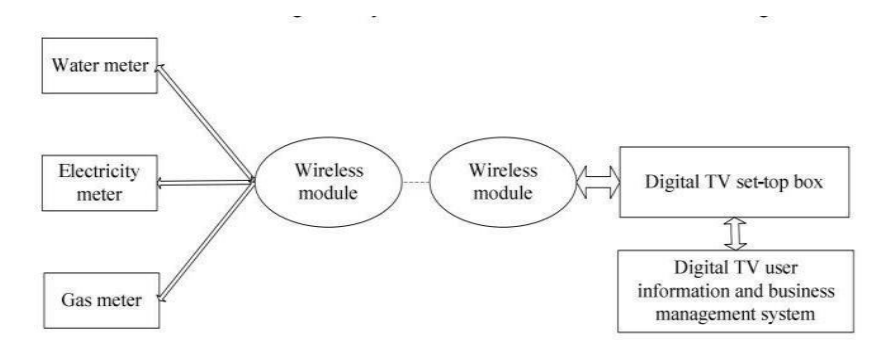

Figure 3.1 Automated Metering wireless module

\subsection{Distributed Power:}

Distributed Power as the name suggest is the technique of distributing the load to increase the efficiency of the system. In Conventional Grid, the electricity was generated at one end and distributed overall but in Smart Grid the idea is taken forward to generate energy at end points also and putting it back into the network which will help a lot in managing the peak loads. Distributed power enables the system to make efficient use of renewable energy sources as solar, wind energy. Distributed Power technique uses two-way communication and two-way power-flow. The concept of microgrid also come into picture when we talk about distributed power generation. Microgrids are smaller smart grids which are independent for their working. Due to microgrids, managing the distribution of power and power generation becomes easier. Distributed Power also talks about the smart electric vehicles. It focuses on future electricity needs which can be achieved without any problems. A consumer can produce energy using solar panels at home and put it back to network or if anyone has excess of energy, they should be able to put it back to the network. Distributed Power creates a solution for peak load problem by dividing the problem and solving on smaller units. 


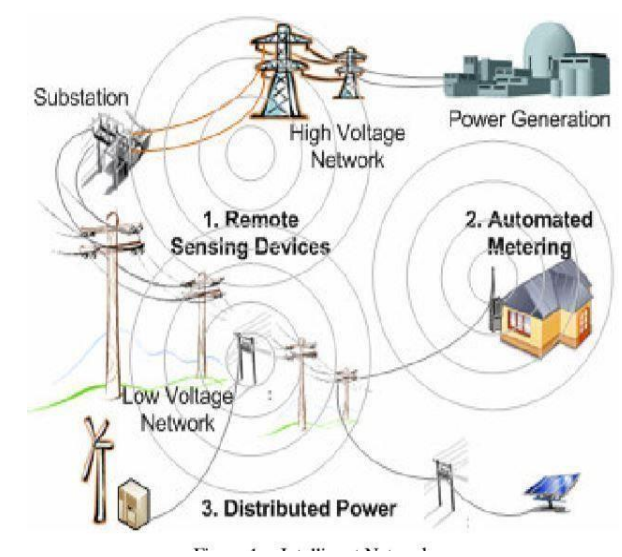

Figure 3.1 Implementing Smart Grid

\subsection{Achieving the Communication using Wireless Network:}

Communication between the devices is necessary for achieving the smart systems. Sensors use light emission and reflection techniques to detect objects without making physical contact with the object which makes it faster as well as dynamic communication. As we have seen in the previous section, sensors can be used for transferring data using wireless light emission technique. For implementing the automated meters, wireless techniques as wi-fi, zig-bee can be used to connect smart devices with the electric meter. Automated metering creates a home area network/personal area network ofsmart devices at home and the meter. It also helps intransferring data from user to generator and generator to user. It helps in providing two-way communication using wireless satellite communication. For the distributed power whenever the energy from any device is needed, the other devices needs to be informed with the requirement data which is achieved best by wireless communication. Unlike the wired structure, wireless structure is easy to implement and manage. Uninterrupted data transfer is the main challenge while using wireless communication which can be used using different security measures we will see in next sections.

\section{Communication in Smart Grid}

\subsection{What Data to be Transferred}

Smart Grid is all about managing the available resources efficiently and being able to handle peak loads properly. The main idea behind Smart Grid will be implemented if the correct data is transferred between consumer, distributor, substations and generators. The communication system should be able transfer real-time data. The data transferred between the system is in digital format. Now the question remains that what data is to be transferred between the system to enable the benefits of Smart Grid. The answer is as follows:1. Sending and Receiving the information about the electricity usage, generation, time consumed, nature of the use and cost required. This information is useful for the customer for managing their consumption and saving the cost. This can be achieved through automated metering and smart devices. 2. Data related to security threads should be transferred to avoid the attacks and threats tosecurity. Also, self-healing devices should be ableto detect the attacks and transfer the data so as to prevent similar attacks on other 
devices. Cyber- Security Attacks can be prevented using this. 3. As we have seen in Chapter 2, Demand and Response data should be transferred between the consumer and generator to manage congestion. 4. Energy Transfer from the user to the network is also important as it creates multiple options of utilizing renewable sources of energy. 5.Data related to unintended events is transferred such as voltage spikes, irrelevant responses, errors while transferring data.

\section{Architecture of Smart grid Wireless Model}

\subsection{General Idea of Architecture and Explanation:}

A Home Area Network (HAN) is a type of network contained within a customer's house that connects customer's appliances and electrical vehicles to a single network. It also contains renewable energy resources and storage equipment to save the generated energy, as well assoftware applications to manage and control all these devices. Appliances are all devices in the house that can beattached to the electricity network, and they include a technology known as Smart Plug (SP), which allow appliances to communicate with other equipment. Anotherimportant device is the Plug-in Electrical Vehicle (PEV). Smart Grid should support the connection of large numberof PEV. So, this is a challenge that must be taken into account while designing a new network. Then, to control this network, the home is equipped with an In-Home Display (IHD). This is an interface between the customer and the HAN. It shows a list of all devices plugged in the network, and statistics about their energy consumption, allowing the user to send command to a specific device (power off), and visualize load equipment data in the home (such as air conditioner(AC), storage battery and EV) and controlling it properly. In addition, wefind the main component in the HAN: Energy Management System (EMS). It controls and optimizes the performance of energy generation, consumption and storage in the HAN. It delivers control commands or events from utilitiesto smart appliances, and gathers all types of information from HAN devices. So, to establish a secure communication connection between utilities and HAN, allHAN customer devices must register themselves firstly to EMS. In order to communicate with utility companies or any other entity that gives energy management services, HAN should be equipped with a gateway. The Energy Services Interface (ESI) plays this role, and routes data between the HAN and the NAN. Generally, the ESI is embedded in the Smart Meter device physically, but, it is logically separate from meter. In fact, the SM collects information about energy usage in customer side, as well as manages control services such as circuit disconnection. It is able to store the metering information internally, and send it to utility via ESI through a two-waycommunication. Multiple HANs can be grouped and forma NAN network. The NAN is the core of the Smart Grid. Itcollects sensed data from customers in a neighbourhood tosend it to an electric utility company after aggregation of the data. Sometimes, the NAN contains field devices suchas intelligent electronic devices (IEDs). In this case the NAN can be called Field Area Network (FAN). The trafficsent by Smart Meters is concentrated at Data Collection Unit (DCU) on the border between HAN and NAN. This equipment transfers the metering information receiving from a set of Smart Meters to equipment whose name is Head EndSystem (HES). This later is a central data collection point for AMI network. It receives data several times a day. Another main system in the NAN is the Meter/Load Controller. It routes commands, requests between the WAN and user side, and it is designed to perform demandresponse service. The last part of Smart Grid architecture is the WAN. It is a network with multiple systems and components connected to each other creating a complex architecture. It covers vast zone from NAN to controlcentre, and provides communication between the electric utility and substations. It also supports real-time monitoring, control and protection applications, which helpdetecting problems in real-time. 


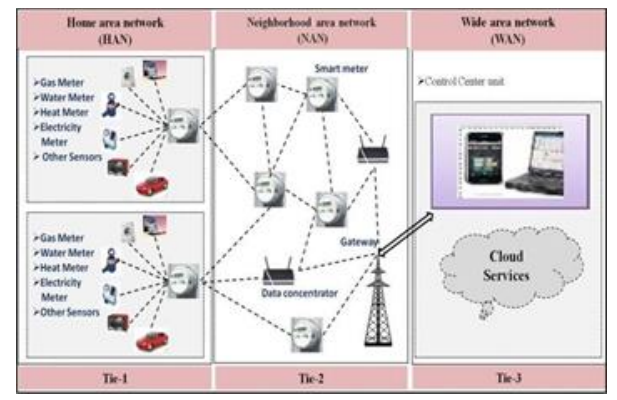

Figure 5.1 General Architecture

\subsection{Flow of Data in Architecture:}

As explained in the architecture, data is transferred from HAN to WAN and WAN to HAN as it is a two-way communication. The NAN acts as a mediator for datatransfer between HAN and WAN. The data flow in Smart Grid is two-way. Each network is connected within itself and to the other network using different wireless protocols. Flow of data/information is WAN to HAN and vice versa in architecture. Different gateways are used to interconnect the network. Also the efficiency of information transfer notonly depends on the distance between the sender and the recipient. Information passed from one node to the other can still get lost somewhere along the way, for example, anode that not cope with the fast data processing. Thetransmission rate of information depends on many parameters, for example: the network topology, place of data origin, brokering environment, fault tolerance and external interference or partial destruction of the network. The data flow should be secure and connection should be stable for efficiency.

\section{Conclusion}

Smart grid is next generation's grid which will help in many applications and making smart devices and reducingthe cost. Using wireless communication and network in Smart Grid will definitely help in managing the grid in easier way than wired network which is complicated. Alsocost of wiring complicated networks is reduced. Smart Grid will make our daily life more reliable and comfortable. Because of Smart Grid, now we will become smarter. Using wireless technologies, the implementation of Smart Grid covering the whole country will definitely succeed. Some of the problems of security for wireless networks needs to be addressed properly to make the system robust and compatible.

\section{References}

[1] Smart Grid Technology in Power Systems, Davood Mohammadi Souran, Hossein Hoshmandi Safa, Behrooz Gohari Moghadam, Mehran Ghasempour and Parisa Tavakkoli Heravi.

[2] Wireless Networks for the Smart Energy Grid: Application Aware Networks Adrian Clark and Christopher J. Pavlovski.

[3] $7^{\text {th }}$ International Conference on Sustainability in Energy and Buildings Towards a smart grid Communication by A Naamane, N.K 